\title{
Zika virus infection: induction, restriction and evasion of host interferon responses
}

\author{
Trisha R Barnard ${ }^{1}$, Maaran Michael Rajah¹ \& Selena M Sagan*,1 \\ 'Department of Microbiology \& Immunology, McGill University, Montréal, QC, H3A 2B4, Canada \\ * Author for correspondence: selena.sagan@mcgill.ca
}

\begin{abstract}
"The recent emergence of ZIKV is multifactorial and likely includes the expanded geographical range of Aedes sp. mosquitoes, the immunological naivety of human populations outside of historically endemic regions and global travel of infected individuals"
\end{abstract}

First draft submitted: 22 August 2017; Accepted for publication: 24 August 2017; Published online: 17 October 2017

Keywords: immune evasion $\bullet$ interferon $\bullet$ interferon-stimulated genes $\bullet$ zika virus

Zika virus (ZIKV) is an arthropod-borne flavivirus that has become a significant global health concern due to its rapid geographic expansion and association with novel neurological pathogenesis [1]. The recent emergence of ZIKV is multifactorial and likely includes the expanded geographical range of Aedes sp. mosquitoes, the immunological naivety of human populations outside of historically endemic regions and global travel of infected individuals [2]. In addition to these epidemiological consequences of globalization, ZIKV has evolved novel mechanisms to induce disease pathogenesis and evade host immune responses $[1,2]$. Understanding how recently acquired viral polymorphisms influence viral pathogenesis and the host immune response during infection is crucial to further our understanding of ZIKV infection and pathogenesis.

ZIKV is a single-stranded, positive-sense RNA virus that encodes a single polyprotein that is catalytically processed by host and viral proteases into three structural proteins (C, prM and E) and seven nonstructural proteins (NS1, NS2A, NS2B, NS3, NS4A, NS4B and NS5). The structural proteins form the virion particle are the primary targets for the host humoral immune response $[1,2]$. The multifunctional nonstructural proteins have been implicated in polyprotein processing, viral RNA replication, virion assembly, as well as evasion of the innate immune response [1]. Herein, we review recent findings regarding how the interferon (IFN) response restricts ZIKV infection and the ways in which ZIKV is able to overcome IFN signaling to circumvent innate immunity.

\section{Type I IFN stimulated genes restrict ZIKV infection}

Type I IFNs (IFN $\alpha / \beta$ ) are transcriptionally regulated and induced following recognition of pathogen components during infection by various host pattern recognition receptors. Activation of pattern recognition receptors leads to synthesis of type I IFNs that trigger antiviral responses by binding to a common type I IFN receptor. Receptor binding stimulates the JAK kinases, which phosphorylate STAT1 and STAT2, leading to the assembly of the ISGF3 complex, composed of STAT1-STAT2 dimers and IRF9, which translocates to the nucleus and binds to IFN-stimulated response elements in the promoters of interferon stimulated genes (ISGs) to regulate their expression [3,4]. This signaling cascade leads to the transcription of more than 300 ISGs, creating a cellular environment resistant to viral replication. It is well established that type I IFN induction is able to restrict ZIKV infection in cell culture [5-8] and in mouse models [6,9].

Recent investigations have demonstrated that several ISGs are able to restrict ZIKV infection [10]. Specifically, the IFN-induced transmembrane proteins, IFITM2 and IFITM3, have been shown to restrict infection at early stages of the viral life cycle and provide protection against ZIKV-induced cytopathic effects in HeLa cells [10]. Additionally, viperin, another antiviral ISG, is able to restrict replication in human hepatoma cells; and viperin knockout mouse embryonic fibroblast cells produce higher ZIKV titers than wild-type cells [11]. However, viperin induction was comparatively low in human cells of placental or neural origin upon infection, highlighting the cell-specific nature of ZIKV-induced ISG expression, which is likely influenced by both viral suppression as well as differences in host

Future Medicine 
intrinsic factors [11]. Thus, in order to establish an infection, ZIKV must have evolved mechanisms to overcome type I IFN signaling and ISGs.

\section{ZIKV evasion of type I IFN signaling}

Several groups have now demonstrated that the ZIKV NS5 protein is able to bind to and degrade STAT2 to inhibit type I IFN signaling in multiple cell types [5,6,12]. However, this effect may be cell type-dependent as infection of human primary monocyte-derived dendritic cells resulted in induction of both STAT1 and STAT2 protein expression, but inhibition of their phosphorylation, rather than selective STAT2 degradation [13]. In addition to the NS5 protein, a recent study suggests that the NS2B/3 protein is able to facilitate degradation of the JAK1 kinase in cell culture, resulting in a reduction in STAT1 phosphorylation [7]. Thus, the NS5 protein appears to be a major player in targeting type I IFN signaling. However, further research will be needed to clarify how ZIKV infection modulates STAT1 and STAT2 expression, phosphorylation and degradation in cell types relevant to viral pathogenesis. On top of regulation of type I IFN signaling, the NS1 and NS4B proteins have been shown to contribute to suppression of type I IFN induction through inhibition of RIG-I-like receptor signaling [7]. Thus, ZIKV nonstructural proteins appear to counter antiviral defenses by inhibiting several pathways leading to induction of type I IFNs and ISGs. However, it is likely that future work will reveal additional mechanisms by which ZIKV is able to counter the type I IFN response [4]. Nonetheless, it is clear that ZIKV has evolved several mechanisms to interfere with type I IFN signaling.

\section{Differential regulation of ZIKV replication by type II \& III IFNs}

In addition to the type I IFN response, type II and III IFNs play complementary roles in shaping innate immunity. In epithelial cells, the primary IFN response is type III (IFN $\lambda$ ) and type III IFNs are likely to be of physiological relevance considering that ZIKV must cross several epithelial barriers to infect the developing fetal brain [3]. In accordance with this, constitutive secretion of type III IFNs by placental trophoblasts was demonstrated to provide protection against ZIKV infection of the placenta [14]. Moreover, type III IFN has been shown to protect against neuroinvasion by related flaviviruses by tightening the blood-brain barrier [15]. As the type III IFNs signal through similar pathways as the type I IFNs and activate a similar set of ISGs, type III IFN induction is likely able to restrict ZIKV infection through analogous ISGs [3]. As such, ZIKV likely evades type I and III IFN signaling cascades using similar mechanisms. Nonetheless, future research into evasion of type III IFN responses is likely to provide useful information on how ZIKV is able to invade the developing fetus and induce neural pathogenesis.

Type II interferon (IFN- $\gamma$ ) is unique in that it can coordinate the transition from innate to adaptive immune responses by assisting in macrophage activation and recruitment of other immune cells to the site of infection [16]. The expression of type II IFNs is limited to cells of the immune system; however, the IFN- $\gamma$ receptor is widely expressed, so most cell types are able to respond to IFN- $\gamma$ [3]. IFN- $\gamma$ signaling initiates upon receptor binding and results in activation of JAK kinases, which subsequently phosphorylate STAT1. Homodimers of phosphorylated STAT1 translocate to the nucleus, where they bind IFN- $\gamma$ activation site elements and stimulate transcription of IFN- $\gamma$-stimulated genes. In contrast to type I and III IFNs, which are able to restrict ZIKV infection, recent findings suggest that type II IFN signaling enhances ZIKV replication in placental and glioblastoma cell lines [5]. Interestingly, ZIKV infection was also demonstrated to induce the expression of many IFN- $\gamma$-stimulated genes; likely due to NS5-mediated depletion of STAT2, effectively shifting the STAT1-STAT2 balance toward STAT1 and resulting in more STAT1 homodimers available to induce transcription of IFN- $\gamma$-stimulated genes [5]. However, the proviral effects of type II IFN signaling is likely cell-type-specific, since other groups have shown that pretreatment with IFN- $\gamma$ restricts ZIKV replication in both lung carcinoma and human foreskin fibroblast cells $[6,8]$. Thus, the interplay between IFN- $\gamma$ and ZIKV replication should therefore be further clarified, as IFN- $\gamma$ is likely to play an important role in the development of ZIKV immunity and pathogenesis.

\section{Cell-type \& strain-specific differences in ZIKV-induced IFN responses}

There is accumulating evidence suggesting cell-intrinsic differences in both induction as well as evasion of IFN signaling during ZIKV infection. Thus, further investigation of cell-type-specific IFN responses could help elucidate the propensity of ZIKV to invade placental and neural tissues. Investigating the importance of IFN signaling in priming recognition of ZIKV-infected cells by the adaptive immune system will also further our understanding of viral infection in the context of the whole organism. Importantly, although the commonly used immunocompro- 
mised murine models provide a useful tool to study viral pathogenesis, immunocompetent models will be important for examination of the host immune response $[1,17]$.

In addition to cell-type-specific differences, recent evidence suggests that there may be strain-specific differences in ISG induction between contemporary and historical ZIKV isolates in both primary human astrocytes and in mouse models of pathogenesis $[2,18]$. However, it remains to be seen whether these strain-specific differences serve to increase the pathogenicity of the virus. Reverse genetic tools will thus be useful to address novel phylogenetic changes acquired by the virus in both cell culture and animal models. Future research will also be needed to identify the specific polymorphisms that mediate these effects.

\section{Conclusion}

In light of the recent epidemic, huge strides have been made towards improving our understanding of ZIKV biology, with several studies helping to elucidate the complex interactions between ZIKV and the IFN response. Future research will help clarify how ZIKV takes on the host immune response and which ISGs play key roles in restricting infection. Furthering our understanding of virus-host interactions will provide valuable insights into ZIKV biology, evolution and mechanisms of host restriction and this information will ultimately aid the development of effective vaccine and antiviral strategies to prevent and combat ZIKV infection.

\section{Acknowledgements}

TR Barnard would like to thank the Frederick Banting and Charles Best Canada Graduate Scholarship - Master's for graduate support. MM Rajah would like to thank the McGill University Faculty of Medicine Max E. Binz Fellowship for graduate support.

\section{Financial \& competing interests disclosure}

This work was supported by startup funds from McGill University as well as operating funds from the Fonds de Recherche du Québec Nature et Technologies (\#189120). SM Sagan is a Tier II Canada Research Chair in RNA Biology and Viral Infections. The authors have no other relevant affiliations or financial involvement with any organization or entity with a financial interest in or financial conflict with the subject matter or materials discussed in the manuscript apart from those disclosed.

No writing assistance was utilized in the production of this manuscript.

\section{References}

1 Rajah MM, Pardy RD, Condotta SA, Richer MJ, Sagan SM. Zika virus: emergence, phylogenetics, challenges, and opportunities. ACS Infect. Dis. 2(11), 763-772 (2016).

2 Tripathi S, Balasubramaniam VR, Brown JA et al. A novel Zika virus mouse model reveals strain specific differences in virus pathogenesis and host inflammatory immune responses. PLoS Pathog. 13(3), e1006258 (2017).

3 Schneider WM, Chevillotte MD, Rice CM. Interferon-stimulated genes: a complex web of host defenses. Annu. Rev. Immunol. 32 513-545 (2014).

4 Miorin L, Maestre AM, Fernandez-Sesma A, Garcia-Sastre A. Antagonism of type I interferon by flaviviruses. Biochem. Biophys. Res. Commun. 492(4), 587-596 (2017).

5 Chaudhary V, Yuen KS, Chan JF et al. Selective activation of type ii interferon signaling by Zika virus NS5 protein. J. Virol. 91(14), pii:e00163-17 (2017).

6 Kumar A, Hou S, Airo AM et al. Zika virus inhibits type-I interferon production and downstream signaling. EMBO Rep. 17(12), 1766-1775 (2016).

7 Wu Y, Liu Q, Zhou J et al. Zika virus evades interferon-mediated antiviral response through the co-operation of multiple nonstructural proteins in vitro. Cell Discov. 3, 17006 (2017).

8 Hamel R, Dejarnac O, Wichit S et al. Biology of Zika virus infection in human skin cells. J. Virol. 89(17), 8880-8896 (2015).

9 Lazear HM, Govero J, Smith AM et al. A mouse model of Zika virus pathogenesis. Cell Host Microbe 19(5), 720-730 (2016).

10 Savidis G, Perreira JM, Portmann JM et al. The IFITMs inhibit Zika virus replication. Cell Rep. 15(11), 2323-2330 (2016).

11 Van Der Hoek KH, Eyre NS, Shue B et al. Viperin is an important host restriction factor in control of Zika virus infection. Sci. Rep. 7(1), 4475 (2017).

12 Grant A, Ponia SS, Tripathi S et al. Zika virus targets human STAT2 to inhibit type i interferon signaling. Cell Host Microbe 19(6), 882-890 (2016).

13 Bowen JR, Quicke KM, Maddur MS et al. Zika virus antagonizes type i interferon responses during infection of human dendritic cells. PLoS Pathog. 13(2), e1006164 (2017). 


\section{Editorial Barnard, Rajah \& Sagan}

14 Bayer A, Lennemann NJ, Ouyang Y et al. Type III interferons produced by human placental trophoblasts confer protection against Zika virus infection. Cell Host Microbe 19(5), 705-712 (2016).

15 Lazear HM, Daniels BP, Pinto AK et al. Interferon-lambda restricts West Nile virus neuroinvasion by tightening the blood-brain barrier. Sci. Transl. Med. 7(284), 284ra259 (2015).

16 Schroder K, Hertzog PJ, Ravasi T, Hume DA. Interferon-gamma: an overview of signals, mechanisms and functions. J. Leukoc. Biol. 75(2), 163-189 (2004).

17 Pardy RD, Rajah MM, Condotta SA, Taylor NG, Sagan SM, Richer MJ. Analysis of the T cell response to Zika virus and identification of a novel CD8 ${ }^{+} \mathrm{T}$ cell epitope in immunocompetent mice. PLoS Pathog. 13(2), e1006184 (2017).

18 Hamel R, Ferraris $\mathrm{P}$, Wichit $\mathrm{S}$ et al. African and Asian Zika virus strains differentially induce early antiviral responses in primary human astrocytes. Infect. Genet. Evol. 49, 134-137 (2017). 